\title{
BEAM LOADING EFFECTS ON HIGH GRADIENT MA-LOADED CAVITY
}

\author{
M. Yamamoto*, M. Fujieda, Y. Hashimoto, Y. Mori, R. Muramatsu, \\ C. Ohmori, Y. Sato, A. Takagi, T. Uesugi, M. Yoshii KEK-Tanashi, Japan
}

\begin{abstract}
We studied the beam loading effects on the High Gradient Cavity(HGC) loaded with Magnetic Alloy(MA) by multiparticle tracking simulation. The Q-Value of the cavity is variable from 0.6 to more than 10 . From the view point of multi-bunch effect, it was found that either very high Q-value or low Q-value was preferable to suppress the periodic transient beam loading. The experimental results of the beam loading compesation using an electron beam are also described.
\end{abstract}

\section{INTRODUCTION}

We have developed a HGC loaded with MA and achieved the high accelerating field gradient of $50 \mathrm{kV} / \mathrm{m}$ [1]. The HGC has some advantages such as the variable Q-value, high stability at the large rf amplitude and realizing low total impedance. Because of these merits, the aspects on the beam loadings are different from that of ordinary ferriteloaded cavity especially on the transient effect and bucket distortion.

So far, small $R / Q$ of the cavity, that is, increasing the Qvalue has been prefered to cure the transient beam loading effect. However the particle tracking simulation shows that the transient effect becomes only a single bunch problem in the low-Q cavity, while it is a multi-bunch effect in the high-Q one. For the HGC, there are two choices to suppress the transient effect because the Q-value of the MA can be changed using radial cut core [2].

On the single bunch effect, the beam-induced voltage is composed of both fundamental frequency and higher harmonics in the low-Q cavity although the high-Q cavity has only fundamental component. However the higher hamonics can be quickly controlled in the low-Q cavity because of the broad-band impedance. In order to study the beam loading effect, an electron-gun and one-path beam line were prepared. Then, the beam-induced voltages up to 3rd higher harmonics were significantly compensated by the beam signal feedback method.

\section{PERIODIC TRANSIENT BEAM LOADING}

In the previous paper [3], the results of the particle tracking simulation under the periodic transient beam loading were shown, and it was found that very low-Q or very high-Q were preferable to avoid the periodic transient beam

\footnotetext{
*E-mail: masanobu@tanashi.kek.jp
}

loading. It is seemed that the transient effect is most severe around $Q \sim h / 2$. It is understood qualitatively that when the cavity damping time is near the revolution frequency, that is, $2 Q / h \omega_{\text {rev }} \sim 1 / \omega_{\text {rev }}$, then the difference of the wake field on each bunch will be largest. It means $Q \sim h / 2$.

The simulation results under the periodic transient beam loading were shown in [3], where 8 of 17 buckets were occupied by the bunch and the others were empty. Simulations for 4 bunches in the 10 buckets and 2 bunches in the 5 buckets were newly performed in the same ring parameters except the harmonic number. Number of the particle per bunch is same in all $Q$ value, $1.25 \times 10^{13}$, and cavity shunt impedance is $6 \mathrm{k} \Omega$.

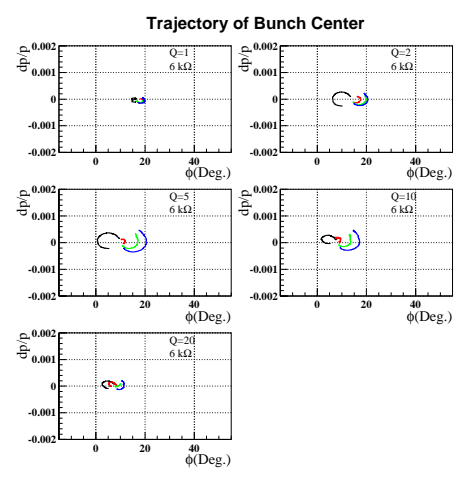

Figure 1: Trajectory of the bunch center in the longitudinal phase space (harmonic number 10, 4 bunches).

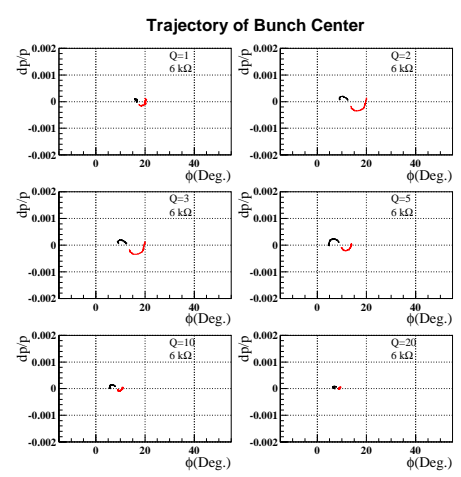

Figure 2: Trajectory of the bunch center in the longitudinal phase space (harmonic number 5, 2 bunches).

In Fig. 1 and 2, each line shows a trajectory of a bunch center in the longitudinal phase space. Vertical axis and horizontal axis are momentum difference and the phase 
measured from the nominal synchronous particle, respectively.

Each bunch is oscillated apart from a locked phase of the $\Delta \phi$-feedback by the transient effect. In case of $h=10$, increasing $Q$ up to 5, the oscillation of each bunch center becomes larger. And increasing $Q$ further, the oscillation becomes smaller again. In the same manner, the oscillation becomes largest around $Q=2 \sim 3$ in case of $h=5$.

In the longitudinal motions of multi-bunches including the energy loss by the wake field, the arrival time of $m$-th bunch on the cavity, $\hat{\tau_{m}}$, is expressed by the perturbative theory as;

$$
\hat{\tau_{m}}=\tilde{\tau_{m}}+\tau_{m}
$$

where $\tau_{m}$ and $\tilde{\tau_{m}}$ are a perturbation and an equilibrium part, respectively. For the equilibrium part, $\tilde{\tau_{m}}$ is defined that the energy gain of the bunch by the cavity gap voltage is equal to the energy loss by the wake field;

$$
e V_{0} \sin \left(\omega_{\mathrm{rf}} \tilde{\tau_{m}}\right)=\sum W(t)
$$

where $W(t)$ is the wake function, and the summation is performed for the past turns and all bunches. In the periodic transient beam loading, $\tilde{\tau_{m}}$ becomes different value on each bunch.

In order to calculate $\tilde{\tau_{m}}$ simply even under the periodic transient beam loading condition, the assumption which the wake field affects only one turn is employed, and resonant type impedance is assumed. Figure 3 shows the calculation result of Eq. (2) in the case of 2 bunches $m=0,1$ filled in the 4 buckets.

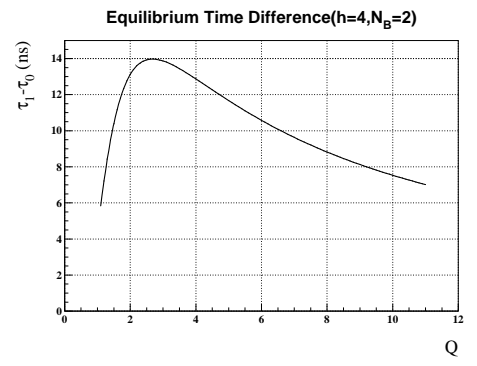

Figure 3: Difference of the equilibrium time $\tilde{\tau_{m}}$ in the case of 2 bunches in the 4 buckets.

The difference between the equilibrium time $\tilde{\tau}_{0}$ and $\tilde{\tau}_{1}$ is largest around $Q \sim 2.5$, and it is near the suggestion by the simulation, $Q \sim h / 2$.

\section{THE EXPERIMENT OF BEAM LOADING COMPENSATION}

In order to investigate the beam loading effects on the HGC, the beam test using electron has been performed. The detail of the electron gun and its setup are described in [4]. In the low-Q cavity, since the bucket distortion by the higher hamonics of the beam-induced voltage is severe problem, the beam loading compensation by the beam signal feedback has been considered.
The beam signal picked up by a Fast Current Transformer is attenuated and delayed by cable line arbitrary, then fed into the cavity through amplifiers. When the attenuation and delay can be optimized, it is expected that the wake voltage is compensated clearly.

The simple scheme of the beam loading compensation was already demonstrated using the electron beam, where the beam signal was simply returned to the amplifier, and it was effective for compensating the one component of the beam-induced voltage [3]. In order to compensate the higher harmonics of the beam-induced voltage as well, the beam signal was separated into each harmonic and the adjustment were done for them, then each signal were combined and fed into the cavity. The setup of the filtering the beam signal is shown in Fig. 4. In this experiment, up to the 3rd higher harmonics of the beam signal were returned to the cavity.

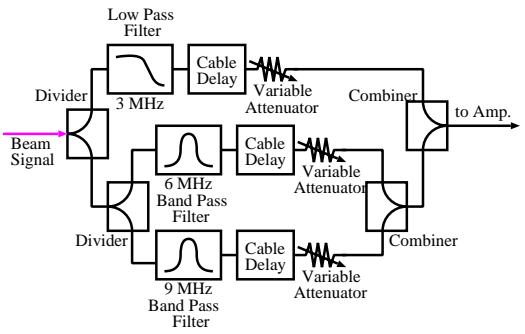

Figure 4: The setup of filtering the beam signal.

The measured voltage of the cavity gap without compensation and with compensation are shown in Fig. 5-(a) and -(b), respectively, and frequency spectrum is shown in Fig. 6, white bar is the spectrum without compensation, and black one is the spectrum with compensation. In this Fourier analysis, ten bunches were counted by a period. As clearly seen in Fig. 6, the fundamental component of the gap voltage became about one hundredth. The second and the third higher harmonic also became one tenth and one fourth, respectively.

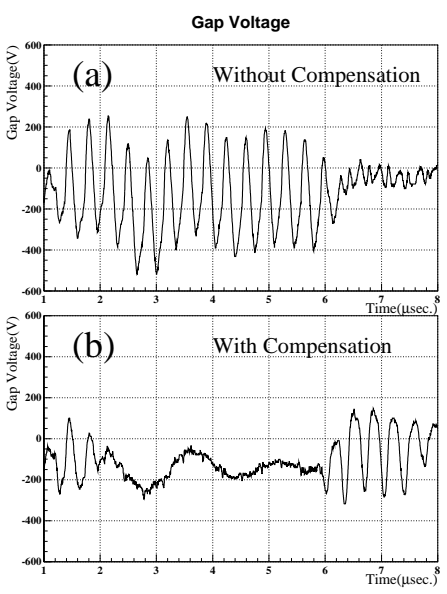

Figure 5: The measured gap voltage with compensation and without compensation. 


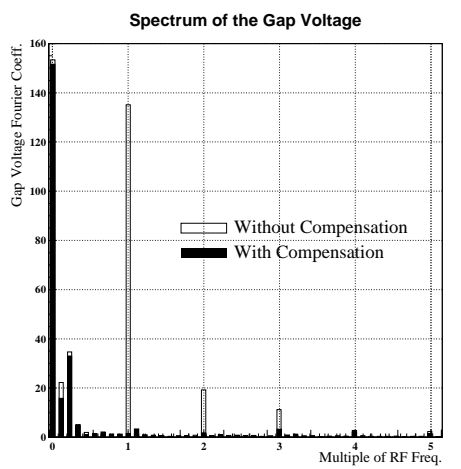

Figure 6: The spectrum of the gap voltage.

In order to study the transient effect, the beam which has some missing bunches is prepared. the test will be done by the variable-Q HGC.

\section{SUMMARY}

Beam loading effects on HGC were investigated, especially for the periodic transient beam loading. From the results of particle tracking simulation and analytic calculation, it was found that the Q-value around a half of a harmonic number should be avoided.

The experiment of the beam loading compesation by the beam signal feedback were done with filtering the beam signal, then three harmonics of the beam-induced voltage were cleary compensated.

\section{REFERENCES}

[1] C. Ohmori et al., ' High Field-Gradient Cavities Loaded with Magnetic Alloys for Synchrotrons, in this conf., 1999

[2] Y. Mori et al., 'A New Type of RF Cavity for High Intensity Proton Synchrotron using High Permeability Magnetic Alloy', p299-p301, Proc. of EPAC98, 1998

[3] M. Yamamoto et al., 'Beam Loading Effects in JHF Synchrotron', Proc. of APAC98, p399-402, 1998

[4] Y. Hashimoto et al., 'Beam Loading Experiment with Short Bunched Electron Beams for New Type of Accelerating RF System of High Intensity Proton Synchrotron, Proc. of EPAC98, p1770-1772, 1998 